\title{
Apoio Social, Transtorno Mental Comum e Uso Abusivo de Álcool em Assentamentos Rurais
}

\author{
Joao Paulo Macedo*, 1 \\ Orcid.org/0000-0003-4393-8501 \\ Magda Dimenstein ${ }^{2}$ \\ Orcid.org/ 0000-0002-5000-2915 \\ Brisana Índio do Brasil de Macêdo Silva ${ }^{1}$ \\ Orcid.org/0000-0003-1150-8291 \\ Hédina Rodrigues de Sousa ${ }^{1}$ \\ Orcid.org/0000-0002-6080-2828 \\ Ana Paula Almeida da Costa ${ }^{\mathbf{1}}$ \\ Orcid.org/0000-0002-0490-7369
}

${ }^{1}$ Universidade Federal do Piauí, Teresina, PI, Brasil

${ }^{2}$ Universidade Federal do Rio Grande do Norte, Natal, RN, Brasil

\section{Resumo}

A população rural brasileira sofre com inúmeras situações de vulnerabilidade, convive com precárias condições de vida e dificuldades de acesso à saúde. Objetivamos investigar as condições de vida e formas de apoio social em assentamentos de reforma agrária no Piaú frente aos casos de Transtorno Mental Comum (TMC) e de uso abusivo de álcool. Para isso, realizou-se um estudo de corte transversal em dois assentamentos: Santo Antônio $(n=1.060)$ e Canárias $(n=432)$, totalizando 1.492 participantes. Foram utilizados questionário sócio demográfico; Self-Reporting Questionnaire (SRQ-20), Alcohol Use Disorders Identification Test (AUDIT) e Escala Medical Outcomes Study (MOS). Dentre os participantes que atenderam aos critérios de inclusão/exclusão para aplicação dos instrumentos $(n=762)$, identificouse $160(21 \%)$ casos de TMC nos dois assentamentos, com maior prevalência entre as mulheres e em moradores acima de 30 anos; e 89 (11,6\%) casos com consumo de risco, uso nocivo e dependente de álcool. Quanto ao apoio social, os moradores o consideram insuficiente e pouco presente no dia a dia. A percepção de fragilidade de suporte nas cinco dimensões avaliadas (material, informação, afetivo, emocional e interação social positiva) está associada aos problemas de infraestrutura, de condições materiais de vida e apresenta diferenças importantes entre homens e mulheres.

Palavras-chave: Apoio social, transtornos mentais comuns, álcool, população rural.

\footnotetext{
Endereço para correspondência: Universidade Federal do Piauí, Campus Universitário Ministro Petrônio Portella, Bairro Ininga, Teresina, PI, Brasil. E-mail: jpmacedo@ufpi.edu.br, mgdimenstein@gmail.com, brisanaindio@gmail.com, hedina_sousa@hotmail.com e ana_almeidac@hotmail.com Financiamento: Conselho Nacional de Desenvolvimento Científico e Tecnológico (CNPq).
} 


\title{
Social Support, Common Mental Disorder and Abusive Use of Alcohol in Rural Settlements
}

\begin{abstract}
Rural Brazilian population suffers with several situations of vulnerability, living in poor life conditions and with difficult access to healthcare. This study aimed to investigate life conditions and forms of social support in rural settlements in Piauí, regarding cases of Common Mental Disorder (CMD) and abusive use of alcohol. Therefore, a cross sectional study was conducted in two settlements: Santo Antonio $(n=1,060)$ and Canárias $(n=432)$, totalising 1,492 participants. The instruments used were a socialdemographic questionnaire; the Self-Reporting Questionnaire (SRQ-20), the Alcohol Use Disorders Identification Test (AUDIT) and the Medical Outcomes Study (MOS). Among the participants who fitted the inclusion and exclusion criteria to participate in the study $(n=762)$, it was identified $160(21 \%)$ cases of CMD in the two settlements, with higher prevalence among women and people over 30 years old; and 89 (11.6\%) of risky consume, harmful use and alcohol dependency. Regarding social support, participants considered insufficient and not enough present in the daily life. The perception of fragility of support in the five evaluated dimensions (material, information, affective, emotional and positive social interaction) is associated with problems in the infrastructure, and in life conditions and it presents important differences between men and women.
\end{abstract}

Keywords: Social support, common mental disorders, alcohol, rural population.

\section{Apoyo social, Trastorno Mental Común y Uso Abusivo de Alcohol en los Asentamientos Rurales}

\section{Resumen}

La población rural brasileña sufre de numerosas situaciones de vulnerabilidad, precarias condiciones de vida y dificultades de acceso a la salud. Se objetivo investigar las condiciones de vida y formas de apoyo social en asentamientos de reforma agraria en Piauí frente a los casos de Trastorno Mental Común (TMC) y de uso abusivo de alcohol. Para tal se realizó un estudio de corte transversal en dos asentamientos: Santo Antônio $(n=1.060)$ y Canárias $(n=432)$, totalizando 1.492 participantes. Se utilizaron un cuestionario demográfico; el Self-Reporting Questionnaire (SRQ-20), el Alcohol Use Disorders Identification Test (AUDIT) y la Escala Medical Outcomes Study (MOS). Dentre los participantes que atenderon a los critérios de inclusión/exclusión para aplicación de los instrumentos $(n=762)$, se identificó $160(21 \%)$ casos de TMC en los dos asentamientos, con prevalencia entre las mujeres y en los residentes mayores de 30 años; y 89 (11,6\%) casos con consumo de riesgo, uso nocivo y dependente de alcohol. En cuanto al apoyo social, los residentes lo consideran insuficiente y poco presente en el cotidiano. La percepción de la fragilidad de suporte en las cinco dimensiones evaluadas (material, información, afectivo, emocional y interación social positiva) se asocia con los problemas de infraestructura, condiciones materiales de vida y presenta diferencias importantes entre hombres y mujeres.

Palabras clave: Apoyo social, trastornos mentales comunes, alcohol, población rural.

A população rural brasileira tem como marca histórica um complexo quadro de desigualdades e dificuldades de acesso às mais diversas políticas públicas. Estudos apontam que as precárias condições de vida das populações do cam- po favorecem o aumento da morbimortalidade de doenças transmissíveis, mortalidade infantil, incidência de endemias e o incremento de doenças crônico-degenerativas (Sparovek, 2003). Por outro lado, a falta de infraestrutura e os proble- 
mas típicos da falta de desenvolvimento social, acompanhado dos altos índices de pobreza e miséria, bem como a precariedade das condições de trabalho e educação, trazem impactos à saúde mental da população do campo, que apesar de evidentes, são poucos os estudos que abordam a temática em nível nacional e internacional (Dimenstein, Leite, Macedo, \& Dantas, 2016; Santana, 2015).

Rehm e Parry (2009), em estudo realizado em âmbito mundial, assinalam para o fato de que os transtornos mentais e o uso abusivo de álcool têm maior impacto negativo em estratos populacionais mais desfavorecidos socioeconomicamente, devido às situações de vulnerabilidade em que se encontram e aos poucos recursos sanitários com que contam. O quadro descrito pelos autores acima ganha maior ênfase quando tratamos de realidades que envolvem áreas de assentamentos rurais no Brasil, pelas duras condições de vida, de trabalho, pobreza, isolamento social e baixa cobertura de serviços públicos que dispõem. Sob este contexto, a população rural fica mais vulnerável à emergência de quadros suscetíveis ao desenvolvimento de Transtornos Mentais Comuns (TMC) e agravos quanto ao consumo abusivo de bebidas alcoólicas (Costa \& Ludermir, 2005; Silva, 2015).

Nesse sentido, é possível observar que saúde e bem-estar são condicionados aos lugares e ambientes de vida das populações, seja no espaço urbano ou espaço rural. Para Loureiro, Lima, Partidário, e Santana (2015), as características dos territórios onde se nasce, cresce, vive, trabalha e envelhece são determinantes para a promoção de saúde e bem-estar, e podem produzir impactos positivos e negativos na saúde mental. Com base neste pressuposto, os autores elencam algumas características a serem levadas em consideração, intituladas "Condicionantes Contextuais da Saúde Mental” (p. 13), divididas em três eixos: ambiente físico e construído (habitação, saneamento, poluição, transporte, espaço verdes, qualidade ambiental, concentração da população, desenho e manutenção do espaço, uso do solo, acesso a equipamentos e serviços, segurança); ambiente socioeconômico (pobreza, rendimento, emprego/desemprego, educação, segurança ali- mentar) e ambiente de interação social e cultural (redes sociais e de apoio social, capital social, coesão social, identidade e pertença, racismo e discriminação, justiça). Logo, consideramos que o apoio social (AS) constitui importante fator de proteção e de promoção da saúde mental.

Apesar das várias definições e concepções do que seja apoio social, de acordo com a literatura, trata-se de um processo multidimensional que desempenha um papel importante na determinação ou na mediação dos/nos níveis de saúde e bem-estar dos indivíduos, envolvendo práticas de sociabilidade e de cuidado produzidos em espaços com a família, amigos, vizinhos, grupos, associações, equipamentos sociais, instituições, etc. (Peña, 2005).

Inúmeros estudos que tratam sobre AS apontam sua importância como fator eficaz na proteção e promoção da saúde, envolvendo aspectos físicos, emocionais e psicoafetivos da vida dos sujeitos, e de forma destacada na vida daqueles que possuem alguma enfermidade crônica (Abreu-Rodrigues \& Seidl, 2013; Rodrigues, Ferreira, \& Caliri, 2013; Santos, 2015). Essa percepção do apoio recebido é referido por Jussani, Serafim, e Marcon (2007) como primordial para manutenção do bem-estar subjetivo das pessoas frente às mais diversas situações de sofrimento psíquico e distúrbios psiquiátricos, depressão, suicídio, problemas nervosos, insônia, enfrentamento de situações estressantes, situações de abstinência de álcool e outras drogas, tratamento de doenças como câncer, socialização da criança na escola, reações frente à hospitalização, gravidez, velhice, dentre outros.

Rocha, Guimarães, e Cunha (2012) indicam que embora o AS seja um importante fator de manutenção e fortalecimento da saúde e do bem-estar, nas classes sociais mais desfavorecidas, o mesmo apresenta-se também como fator de mediação, reposicionamento subjetivo e enfrentamentos em relação às dificuldades de acesso e de tratamento nos serviços públicos de saúde. Entretanto, são poucos os estudos que têm se dedicado ao tema junto à população que vive em contextos rurais.

No Brasil, o estudo de Costa e Ludermir (2005) é um dos poucos que investe sobre a 
relação entre apoio social, saúde mental e contexto rural. Trata-se de uma investigação sobre a prevalência de TMC e sua associação com o apoio social em uma comunidade da Zona da Mata de Pernambuco. Quanto às pesquisas sobre o consumo de álcool e apoio social em áreas rurais, são raros os estudos sobre o tema no país. No entanto, algumas pesquisas apontam que fatores como a presença de suporte social, acesso ao lazer e políticas públicas estão inversamente relacionados ao uso abusivo de álcool em áreas rurais (Ronzani, 2008). Assim, considerando o impacto social, econômico e familiar decorrente dos problemas de saúde mental, além da escassez de estudos que tratam sobre a temática em contextos rurais, desenvolvemos essa pesquisa com o objetivo de investigar as condições de vida e formas de apoio social em assentamentos de reforma agrária no Piaú frente aos casos de TMC e de uso abusivo de álcool.

\section{Método}

Trata-se de um estudo de corte transversal, de caráter descrito-exploratório, realizado em dois assentamentos de reforma agrária do estado do Piauí: Santo Antônio do Campo Verde situado no município de Sigefredo Pacheco e a Reserva Extrativista da Marinha do Delta do Parnaíba (RESEX) localizada no norte do Estado. Participaram do estudo todos os moradores maiores de 18 anos dos dois assentamentos, constituindo-se como critérios de exclusão a recusa em participar da pesquisa e a existência de condição médica ou psicológica que impossibilitasse a participação. Os participantes foram entrevistados em seus domicílios. Dentre as ferramentas para coleta dos dados foram utilizados: (a) Questionário Social Demográfico Ambiental (QSDA), versão modificada do material elaborado pelo Departamento de Geologia da Universidade Federal do Rio Grande do Norte (UFRN), organizado em torno dos seguintes aspectos: renda, ocupação, escolaridade, número de moradores residentes, idade, sexo, religião, infraestrutura dos assentamentos, condições de saúde, acesso aos serviços públicos de saúde, bem como aos programas de transferência de renda, crédito e assistência rurais. O questionário original abordava ainda questões relacionadas a realidade fundiária, detalhamento da infraestrutura da moradia, produção agrícola, maquinário, insumos, dentre outros, que não foram objeto desta investigação, além de termos atualizado informações sobre as políticas sociais. (b) Self-Reporting Questionnaire (SRQ-20) para a detecção dos casos de TMC. É um questionário validado no Brasil (Mari \& Williams, 1986) e recomendado pela Organização Mundial da Saúde (OMS) para estudos comunitários e na Atenção Primária à Saúde (AP). É composto por 20 itens, com possibilidade de resposta do tipo sim/não. Os escores obtidos sinalizam a probabilidade de presença de TMC ou desconforto emocional (construto avaliado), variando de 0 (nenhuma probabilidade) a 20 (extrema probabilidade). Adotou-se como ponto de corte o quantitativo de 07 ou mais respostas positivas como indicativo de TMC, com base em estudos que tratam sobre usuários de serviços de atenção primária no Brasil (Gonçalves, Stein, \& Kapczinski, 2008). (c) Alcohol Use Disorders Identification Test (AUDIT) para a detecção dos casos de uso de álcool. Trata-se de questionário validado no Brasil (Babor, Higgins-Biddle, Saunders, \& Monteiro, 2001) e recomendado pela OMS para detecção do consumo excessivo de álcool e apoio à intervenção breve pelos profissionais da AP. Contêm dez questões sobre consumo e sintomas relacionados ao uso problemático de álcool nos últimos 12 meses. Adotou-se como ponto de corte o quantitativo de 08 ou mais itens pontuados, classificados em consumo de risco, nocivo ou de dependência, conforme estabelecido pelo instrumento. (d) Medical Outcomes Study (MOS-SSS) para os casos em que o entrevistado tenha pontuado no SRQ-20 e/ou no AUDIT. Trata-se da Escala de Apoio Social desenvolvida por Sherbourne e Stewart (1991) e validada no Brasil por Griep, Chor, Faerstein, Werneck, e Lopes (2005). Enquanto construto foi elaborado e subdivido em cinco dimensões, a saber: Apoio Material (disponibilidade de recursos e ajuda material); Apoio de Informação (existência de pessoas com quem obter informações e conselhos); Apoio Afetivo (pessoas que demonstram amor e afeto); Apoio Emocional 
(externalização de confiança, escuta e interesse); e o Apoio de Interação Social Positivo (como a pessoa se relaciona e dispõe de companhia para se divertir e relaxar; Griep et al., 2005).O MOS-SSS é composto por 19 questões. Para cada resposta, foram atribuídos escores variando de 1 (nunca) a 5 (sempre). Foram estabelecidos dois grupos para cada tipo de apoio, considerados como baixo e alto apoio social, com base na mediana estatística dos dados em cada dimensão.

Sobre o uso das ferramentas acima referidas, apesar do esforço de alguns poucos autores em tentar validá-las em populações rurais (Costa \& Ludermir, 2005; Moretti-Pires \& Corradi-Webster, 2011), entendemos que as mesmas foram originalmente elaboradas com base em populações urbanas e que a sua aplicação em meios rurais precisa considerar o contexto e as singularidades que marcam a história de vida dessas pessoas.

A análise dos dados, com base no Software Statistical Package for the Social Sciences (SPSS) for Windows, versão 20, utilizou a frequência absoluta, relativa e mediana (valor mínimo e máximo) e para comparação entre grupos recorreu-se ao teste qui-quadrado de Pearson, considerando como nível de significância o valor de 0,05 . Por fim, foram cumpridos os procedimentos éticos da Resolução do Conselho Nacional de Saúde de $n^{\circ} 466 / 12$, com CAAE $\mathrm{N}^{\mathrm{o}}$ 54297716.6.0000.5214 no Comitê de Ética de Pesquisa da Universidade Federal do Piauí (UFPI), tendo todos os participantes assinado o Termo de Consentimento.

\section{Resultados e Discussões}

\section{Caracterização Sócio Demográfica dos Assentamentos e das Familias Pesquisadas}

O Piauí, que conta com uma população de 3.118.360 habitantes, distribuída em 224 municípios $\left(12,4 \mathrm{hab} / \mathrm{km}^{2}\right)$, possui $34,2 \%$ da população vivendo em áreas rurais. O índice nacional é de 15,6\% (Instituto Brasileiro de Geografia Estatística [IBGE], 2010). Conta com a segunda pior renda por domicílio do país ( $\mathrm{R} \$ 1.354)$. Nos últimos dez anos, metade da população que se encontrava em situação de extrema pobreza (total de 800 mil) teve suas condições de vida melhoradas. Dentre os principais elementos que têm colaborado para o aumento e distribuição da renda está a adoção de políticas de transferência de renda, aumento do salário-mínimo e do crédito. Ademais, apresenta uma das mais altas taxas $(21,10 \%)$ de analfabetismo do Brasil, sendo estes índices maiores nas áreas rurais devido à dificuldade de acesso à rede escolar e a manutenção do trabalho infantil (Programa das Nações Unidas para o Desenvolvimento [PNUD], 2010). De acordo com o Instituto Nacional de Colonização e Reforma Agrária (INCRA), há no Piauí 31.193 famílias que vivem em 497 assentamentos rurais.

Circunscrevemos o estudo, conforme mencionado anteriormente, em dois assentamentos de reforma agrária: um localizado no território "Planície Litorânea", conhecido como Reserva Extrativista da Marinha do Delta do Parnaíba (RESEX), mais especificamente na Comunidade Canárias; outro situado no território "Carnaubais", conhecido como Santo Antônio do Campo Verde, localizado no município de Sigefredo Pacheco.

A RESEX fica localizada no Delta das Américas. Apesar de vinculada administrativamente ao município de Araioses no Maranhão, o INCRA a considera como área de assentamento pertencente ao estado do Piauí. O assentamento é composto por um aglomerado de comunidades dispersas em ilhas no Delta do Rio Parnaíba: Ilha das Canárias, Passarinho, Caiçara, Torto e Morro do Meio, tendo toda sua extensão banhada por rios. O acesso é feito por embarcações grandes e pequenas. O Delta do Parnaíba é uma área de proteção ambiental, criada em 2005, gerenciada pelo INCRA e pelo Instituto Chico Mendes de Conservação da Biodiversidade (ICMBio), embora já fosse ocupada há muito tempo pelos moradores.

A coleta de dados na RESEX deteve-se na comunidade das Ilhas das Canárias, em função dos seguintes critérios: grande quantidade populacional (maior ilha do Delta), área de grande vulnerabilidade socioeconômica e facilidade de acesso, se comparado às demais comunidades 
da região. Participaram do estudo 269 famílias, totalizando 1.060 indivíduos. Trata-se uma população composta, em sua maioria, por jovens adultos, solteiros $(53,16 \%)$, com prevalência do sexo masculino (51,51\%), nível de escolaridade ensino fundamental incompleto/completo $(57,79 \%)$ e renda familiar mensal em até $1 / 2$ salário $(54,25 \%)$. Com principais fontes de sustento enumera-se a atividade pesqueira, programas de transferência de renda e aposentadoria.

Quanto ao assentamento Santo Antônio do Campo Verde, localiza-se no centro-oeste do Estado do Piauí, no município de Sigefredo Pacheco, a $8 \mathrm{~km}$ da sede do município. Foi criado por meio das lutas do Movimento dos Sem Terra (MST) em parceria com o Sindicato dos Trabalhadores Rurais, em um período de grande mobilização dos movimentos sociais rurais e sindicais, por se tratar de uma área de grande extensão territorial improdutiva, com cerca de dez mil hectares de terra. No ano de 1996 foi decretada a desapropriação dessa área e em 1997 foi dado início pelo INCRA os primeiros cadastramentos para formação do assentamento. Somente em 1998 começaram a serem construídas as casas.

Participaram do estudo em Santo Antônio do Campo Verde 143 famílias, totalizando 432 indivíduos. A população foi composta predominantemente por jovens adultos, do sexo masculino $(51,62 \%)$, casado $(52,2 \%)$, dado esse que difere de Canárias que é composta, em sua maioria, por pessoas solteiras. Quanto à escolaridade, $56,23 \%$ da população investigada cursaram até o ensino fundamental incompleto/completo. As famílias encontram sustento na agricultura familiar, nos programas de transferência de renda e aposentadoria. A maioria da população assentada tem renda de até $1 / 2$ salário (59,95\%).

No tocante às condições de vida dos assentamentos, a maioria das casas eram de alvenaria, com telhas e piso com cerâmica. $\mathrm{O}$ acesso a água é fornecido pela rede pública, na maioria das casas em Santo Antônio; e através de poço em Canárias. O tratamento da água é feito por filtração ou cloração da água. Quanto aos dejetos, se dá por meio de fossa séptica e o destino do lixo por coleta pública ou queimada. Sobre o acesso aos serviços de saúde, $81,82 \%$ das famílias do assentamento Santo Antônio e 89,14\% em Canárias informaram serem assistidos por Agente Comunitário de Saúde (ACS). No entanto, o que se percebe pelos relatos é que o acompanhamento dos ACS não tem regularidade. Passam meses sem realizar as visitas domiciliares, mostrando uma fragilidade da rede de atenção e cuidado à saúde. No que tange aos atendimentos no posto de saúde, ambos os assentamentos informaram receber atendimento, sendo que no primeiro a unidade de saúde fica na sede do município e no segundo na própria comunidade. As doenças mais comuns, em ambos os assentamentos, são: gripe, pneumonia e demais doenças respiratórias, sarampo, catapora, verminose, diarreia, além de hipertensão e diabetes. E, apesar de Canárias contar com uma unidade da Estratégia Saúde da Família, os níveis de adesão aos programas de saúde são bem mais baixos se comparado com Santo Antônio.

Sobre os programas socais, $75,52 \%$ das famílias são beneficiárias do Programa Bolsa Família em Santo Antônio e 70,63\% em Canárias. Quanto ao crédito instalação, 62,24\% das famílias recebem o auxílio em Santo Antônio, e somente 2,97 \% famílias de Canárias. A mesma situação é vista no Programa Nacional de Fortalecimento da Agricultura Familiar (PRONAF), com o recebimento por $44,06 \%$ famílias de Santo Antônio, enquanto somente $2,60 \%$ das famílias de Canárias recebem. Tais aspectos repercutem diretamente nas condições de moraria e de trabalho e renda nos assentamentos.

Os dados encontrados nos dois assentamentos retratam uma realidade similar encontrada no meio rural do país marcada por condições de vulnerabilidade social, pobreza e miséria. Pois, embora se registre uma melhora nos indicadores sociais, bem como nas condições de moradia, educação, saúde e lazer nas áreas rurais do Brasil, resultado da implantação e expansão de projetos sociais e agrários, ainda é possível observar considerável índice de não alfabetizados, dificuldade no acesso aos serviços públicos e assistência técnica, precariedade das condições de trabalho e dependência dos programas de transferência de renda, falta de estradas, de transpor- 
tes adequados, meios de comunicação, poucos recursos hídricos, destinação adequada do lixo, e manutenção de migração rural-urbana em busca de serviços de saúde, educação, trabalho e renda. Essa foi a realidade encontrada em Santo Antônio e em Canárias.

\section{Transtornos Mentais Comuns e Apoio Social}

Do total de 1.492 indivíduos residentes nos dois assentamentos investigados, aplicou-se o SRQ-20 em 767 indivíduos, sendo 255 em Santo Antônio e 507 em Canárias. Foram descartados 5 questionários por problemas no preenchimento. Do total de questionários válidos, $21 \%$ ficaram acima do ponto de corte, configurando, portanto, possível indicativo de TMC.

Em ambos os assentamentos houve predomínio do sexo feminino $(32,46 \%)$ com sujeitos acima do ponto de corte, enquanto entre os homens o percentual foi menor $(26,17 \%)$. Chama atenção os resultados por assentamento, considerando que o índice de mulheres que pontuou na ferramenta foi mais expressivo em Canárias (72,55\%), se comparado com Santo Antônio (55,17\%), como observar-se na Tabela 1.

Tabela 1

SRQ-20 Homens e Mulheres

\begin{tabular}{|c|c|c|c|c|c|c|}
\hline \multirow[b]{2}{*}{ Santo Antônio } & \multicolumn{2}{|c|}{ Abaixo do ponto de corte } & \multicolumn{2}{|c|}{ Acima do ponto de corte } & \multicolumn{2}{|c|}{ Total (PI) } \\
\hline & $N^{\mathrm{o}}$ & $\%$ & $N^{\mathrm{o}}$ & $\%$ & $N^{\mathrm{o}}$ & $\%$ \\
\hline Masculino & 99 & 50.25 & 26 & 44.83 & 125 & 49.02 \\
\hline Feminino & 98 & 49.75 & 32 & 55.17 & 130 & 50.98 \\
\hline Total & 197 & 100 & 58 & 100 & 255 & 100 \\
\hline \multicolumn{7}{|l|}{ Canárias } \\
\hline Masculino & 189 & 46.67 & 28 & 27.45 & 217 & 42.80 \\
\hline Feminino & 216 & 53.33 & 74 & 72.55 & 290 & 57.20 \\
\hline Total & 405 & 100 & 102 & 100 & 507 & 100 \\
\hline
\end{tabular}

Quando cruzamos os resultados dos que pontuaram acima do ponto de corte com os dados sócio demográficos, na perspectiva de considerar o contexto de vida dessas pessoas, observa-se que além do sexo feminino, esses estão em sua maioria na faixa etária de 30 a 49 anos, nível de escolaridade ensino fundamental incompleto/ completo, estado civil casado, com renda família de até 1 salário mínimo.

Nos poucos trabalhos que tratam especificamente sobre população rural, autores como Dimenstein et al. (2016) sinalizam possível associação entre renda, escolaridade, gênero e maior incidência de TMC, embora não indiquem uma relação determinista e de causalidade. Autores como Costa e Ludermir (2005), Pinho e Araújo (2012) entendem que a maior prevalência de TMC em mulheres que vivem áreas rurais deve- -se a diversos fatores tais como a elevada carga de trabalho doméstico, cuidados com os filhos, baixa gratificação, submissão ao companheiro em relação as decisões da casa, além da falta de visibilidade na realização do trabalho doméstico.

Por outro lado, os atravessamentos de gênero entre homens e mulheres envolvendo o TMC comportam particularidades (Dimenstein et al., 2016). Para esses autores, enquanto o adoecimento dos homens em assentamentos rurais está mais associado à carga do trabalho agrícola, à precariedade das condições de trabalho, à perda da vitalidade física, bem como ao surgimento de doenças crônicas, nas mulheres relaciona-se ao trabalho doméstico, caracterizado pela dupla jornada de trabalho, o número de filhos, a violência de gênero praticada por parceiro íntimo, eventos de vida estressantes como perdas de parentes 
próximos, separação, os quais contribuem para o surgimento ou agravamento do adoecimento mental.

Loureiro et al. (2015) chamam atenção para os determinantes sociais no processo de adoecimento psíquico, elencando as condições de vida e desigualdade social como elementos predisponentes de sofrimento psíquico. Nesse sentido, a realidade socioeconômica dos assentamentos investigados, já apresentados anteriormente, indica dificuldades de acesso aos bens e serviços, como saúde, educação, trabalho, transporte, políticas públicas, saneamento básico, o que pode deixar a população assentada ainda mais vulnerável a situações de adoecimento/sofrimento psíquico.

Ao detalharmos os resultados relacionados às dimensões do SRQ-20, em Santo Antônio, as mulheres se destacam em todas as dimensões do instrumento se comparado aos homens, principalmente quanto aos pensamentos depressivos $(63,08 \%)$, seguido por decréscimo de energia vital $(58,29 \%)$, sintomas somáticos $(57,14 \%)$ e humor depressivo ansioso (46,48\%). Em Canárias, o predomínio das mulheres nas dimensões do SRQ envolve os sintomas somáticos $(63,21 \%)$, decréscimo de energia $(62,9 \%)$, humor depressivo ansioso $(62,47 \%)$, pensamentos depressivos $(56,25 \%)$.

No geral, os dados por dimensão do SRQ, principalmente em Santo Antônio, chamam atenção para o grande percentual de pessoas que pontuou na dimensão de pensamentos depressivos, já que no total de 255 respondentes, $51 \%$ pontuaram nessa categoria. A maioria recai novamente sobre as mulheres, o que não deixa de ser um dado preocupante, tendo em vista o atravessamento de gênero nas formas de expressão do adoecimento, de modo que questões referentes à depressão, transtorno de ansiedade e ideação suicida afetam mais mulheres que homens (Rabasquinho \& Pereira, 2007). Quando indagados sobre pensamentos de acabar com a própria vida, do total da amostra pesquisada nos dois assentamentos, 102 pessoas disseram que "sim", correspondendo $13,4 \%$ dos casos, o que sugere ser um dado expressivo e que inspira preocupação e desafios às políticas de saúde mental. Desse total, 28,4\% residem em Santo Antônio e
$74,6 \%$ em Canárias, com prevalência feminina.

Àqueles que ficaram acima do ponto de corte no SRQ-20 $(n=160)$, aplicou-se o MOS-SSS. No tratamento dos resultados da Escala de Apoio Social (MOS-SSS) foram excluídos cinco questionários, restando 155 ( $\mathrm{AS}=55$ e $\mathrm{CN}=100$ ), ilustrado na tabela a seguir.

Quanto às dimensões acerca do apoio social percebido pelos indivíduos com indicativo de TMC, observa-se na Tabela 2, que há aqueles que estão abaixo da mediana, ou seja, que relatam não dispor de apoio social suficiente; e os que estão acima da mediana dizem contar com algum tipo de apoio social. E dentre as dimensões investigadas nos assentamentos, de modo geral, a maioria do percentual dos entrevistados está abaixo da mediana, sendo na dimensão do Apoio Material (58,06\%), no Apoio Emocional (56,13\%), no Apoio Afetivo (54,19\%) e de Informação (54,19\%). Especificamente quanto à dimensão Apoio de Interação Social Positiva todos os resultados concentraram-se abaixo da mediana, o que pode sinalizar falta de espaços e de momentos de convivência, principalmente no âmbito comunitário, com poucas atividades de lazer, cultura e de interação social nos assentamentos. Desse modo, compreende-se, com base na fragilidade da infraestrutura e condições materiais de vida, bem como da carência de recursos disponíveis nos assentamentos, que tais fatores podem contribuir, de alguma forma, com o motivo pelo qual pessoas com sofrimento psíquico percebem como insuficiente o apoio social recebido nas dimensões investigadas. Tais aspectos poderiam ser aprofundados em futuros estudos com análises estatísticas mais específicas, que extrapolam o caráter exploratório deste estudo.

Considerando o padrão de respostas entre homens e mulheres sobre o AS, observamos que o fator gênero sugere impactar de forma distinta naqueles que apresentaram forte indícios de TMC. De 155 respondentes no MOS-SSS, $68,38 \%$ foram mulheres que sinalizaram contar com baixo apoio em todas as dimensões. Esse é um dado preocupante visto que as mulheres sofrem fortemente com essa desproteção.

Ao cruzar cada dimensão do AS com o perfil das pessoas que pontuaram abaixo do ponto 
Tabela 2

Apoio Social (MOS-SSS) dos Respondentes do SRQ-20

\begin{tabular}{|c|c|c|c|c|c|c|c|}
\hline \multirow{3}{*}{ Dimensão } & & \multicolumn{6}{|c|}{ Assentamentos } \\
\hline & & \multicolumn{2}{|c|}{ Santo Antônio } & \multicolumn{2}{|c|}{ Canárias } & \multicolumn{2}{|c|}{ Total (PI) } \\
\hline & & $N$ & $\%$ & $N$ & $\%$ & $N$ & $\%$ \\
\hline \multirow{3}{*}{ Apoio Material } & Abaixo da mediana (Zero até 95) & 31 & 56.36 & 59 & 59 & 90 & 58.06 \\
\hline & Acima da mediana (acima de 95) & 24 & 43.64 & 41 & 41 & 65 & 41.94 \\
\hline & Total & 55 & 100 & 100 & 100 & 155 & 100 \\
\hline \multirow{3}{*}{ Apoio Afetivo } & Abaixo da mediana (Zero até 95) & 33 & 60 & 51 & 51 & 84 & 54.19 \\
\hline & Acima da mediana (acima de 95) & 22 & 40 & 49 & 49 & 71 & 45.81 \\
\hline & Total & 55 & 100 & 100 & 100 & 155 & 100 \\
\hline \multirow{3}{*}{ Apoio Emocional } & Abaixo da mediana (Zero até 90) & 31 & 56.36 & 56 & 56 & 87 & 56.13 \\
\hline & Acima da mediana (acima de 90) & 24 & 43.64 & 44 & 44 & 68 & 43.87 \\
\hline & Total & 55 & 100 & 100 & 100 & 155 & 100 \\
\hline \multirow{3}{*}{$\begin{array}{l}\text { Apoio } \\
\text { de Informação }\end{array}$} & Abaixo da mediana (Zero até 90) & 31 & 56.36 & 53 & 53 & 84 & 54.19 \\
\hline & Acima da mediana (acima de 90) & 24 & 43.64 & 47 & 47 & 71 & 45.81 \\
\hline & Total & 55 & 100 & 100 & 100 & 155 & 100 \\
\hline \multirow{3}{*}{$\begin{array}{l}\text { Apoio de } \\
\text { Interação } \\
\text { Positiva }\end{array}$} & Abaixo da mediana (Zero até 100) & 55 & 100 & 100 & 100 & 155 & 100 \\
\hline & Acima da mediana (acima de 100) & 0 & 0 & 0 & 0 & 0 & 0 \\
\hline & Total & 55 & 100 & 100 & 100 & 155 & 100 \\
\hline
\end{tabular}

de corte no SRQ-20, observamos algumas especificidades. Em todas as dimensões são as pessoas casadas que parecem contam com menor apoio. No caso das mulheres, o percentual que indicou baixo apoio sugere ser bem mais significativo $(74,1 \%$ no material, $81 \%$ no emocional, $77,4 \%$ no afetivo, $78 \%$ de informação e $79,6 \%$ de interação positiva) do que entre homens casados (53,1\% no material, 51,6\% no emocional, no afetivo e de informação; e 63,5\% de interação positiva). Porém, chama atenção um razoável percentual de homens solteiros nesta condição (28,1\% no material, e $25,8 \%$ no emocional, no afetivo e de informação; e 19,2\% de interação positiva). Entretanto, enquanto há destaque de baixo apoio entre as mulheres que têm filhos (70,7\% no material, $70,7 \%$ no emocional, $75,5 \%$ no afetivo, 64,4\% de informação; e 70,9\% de interação positiva), entre os homens o destaque é para aqueles que não têm filhos $(50 \%$ no material, $58,1 \%$ no emocional, no afetivo e de informação; e 53,8\% de interação positiva), indicando possível sobrecarga das mulheres com os filhos e a ausência de vida familiar como elementos associados ao sofrimento de mulheres e homens.

Quanto à ocupação, as mulheres que percebem baixo apoio são donas de casa que desenvolvem paralelamente atividades na roça e na pesca $(67,2 \%$ no material, $72,4 \%$ no emocional, $73,6 \%$ no afetivo, $64,4 \%$ de informação e $70,9 \%$ de interação positiva). Os homens são agricultores e pescadores $(65,6 \%$ no material, $58,1 \%$ no emocional, $58 \%$ no afetivo, $58,1 \%$ de informação e $63,5 \%$ de interação positiva). Quanto à escolaridade, as mulheres que contam com menor apoio têm mais escolaridade que os homens. As mulheres nesta condição possuem o ensino fundamental (48,3\% no material, $58,6 \%$ no emocional, $56,6 \%$ no afetivo, $57,6 \%$ de informação e $56,3 \%$ de interação social), enquanto os homens são em maior número analfabetos $(53,1 \%$ no 
material, $64,5 \%$ no emocional, $63,5 \%$ no afetivo, $64,5 \%$ de informação e $53,8 \%$ de interação social). Quanto à renda, tanto homens quanto mulheres com menor apoio estão no grupo de até $1 / 2$ salários mínimos, apesar de que o percentual de mulheres $(55,10 \%$ no material, $63,80 \%$ no emocional, $60,40 \%$ no afetivo e de informação; e $59,20 \%$ interação positiva) se sobressai em relação aos homens em todas as dimensões $(46,90 \%$ no material, $51,60 \%$ no emocional, no afetivo e de informação; e 48,10\% interação positiva).

Em resumo, consideramos que as mulheres parecem vivenciar níveis mais preocupantes de vulnerabilidade nesses assentamentos. O fato de lidarem com elevada carga de trabalho, desdobrando-se entre as atividades de cuidar de casa, dos filhos, do marido, da atividade agrícola ou pesqueira, além de assumirem o papel de cuidadora, que histórica e socialmente lhes é atribuído, tudo isso sugere resultar em intensa sobrecarga física e emocional, traduzidos, possivelmente, pelos sinais e sintomas do TMC. Todo o esforço e sofrimento gerados não são reconhecidos pelo companheiro e filhos, o que certamente pode gerar grande impacto na percepção de apoio social especialmente quanto às dimensões material, emocional e afetiva.

Se tomarmos o Apoio Material como analisador do impacto das relações de gênero sobre o apoio percebido, nota-se que caso as mulheres adoeçam, precisem ir ao médico e/ou necessitem de ajuda para as tarefas diárias e cuidado com os filhos essas relatam contar com pouquíssima ajuda, já que tais funções são tidas como de sua responsabilidade. Diferente dos homens que, comumente, contam com o apoio e ajuda da esposa e dos filhos quando precisam, e se sentem reconhecidos por isso. Ademais, ficou evidenciado, no caso dos homens, que o casamento e ter filhos constitui fator protetivo quanto à oferta de apoio social. Sendo que entre os homens solteiros ou sem filhos houve um maior índice de insatisfação quanto ao apoio recebido. Tais aspectos demandam novas investigações para avaliar possíveis relações entre esses fatores.

Além disso, ainda há uma visão machista e sexista do lugar social da mulher ligado ao lar, que atrelado a ampla sobrecarga de trabalho do- méstico (por vezes invisível e solitário) acabam por afastar as mulheres da vida social do assentamento. As mulheres dos assentamentos relatam contar como poucas pessoas que a demonstrem amor e afeto (Apoio Afetivo), que possam exercer uma relação de confiança, escuta e interesse (Apoio Emocional) e identificam poucas pessoas em quem podem confiar e compartilhar suas preocupações e medos ou até mesmo com quem possa se divertir (Apoio de Interação Social Positiva), geralmente, as formas de apoio social advêm dos filhos e, em alguns casos, do companheiro. Quanto ao Apoio de Informação, sugere que devido à falta de retaguarda de profissionais de saúde e da existência de fortes laços sociais e comunitários, há fragilidade e insuficiência do apoio percebido, principalmente quanto às redes formais, advindas das políticas de saúde e demais equipamentos de proteção social.

Voltando à Tabela 2, observa-se que no assentamento Canárias, por contarem com infraestrutura e condições de vida mais precarizadas, o Apoio Material sobressai em 59\% dos respondentes estando abaixo da mediana, seguido do Apoio Emocional (56\%), Apoio de Informação (53\%), Apoio Afetivo (51\%) indicando índices insatisfatórios em todas as dimensões. Em Santo Antônio há destaque para o Apoio Afetivo $(60 \%)$, considerando a baixa percepção de relações de confiança, escuta e interesse frente aos problemas e sofrimento. Nas demais dimensões que envolvem o Apoio Material, Emocional e de Informação, também pontuaram abaixo da mediana, figurando como baixo apoio (56.36\%). E quanto a Interação Social Positiva como se é possível observar, em ambos os assentamentos, todos os respondentes apontaram deter de apoio insuficiente, sendo uma dimensão a ser considerada nas estratégias de prevenção e promoção de saúde. A seguir, trataremos sobre os casos de uso de álcool e apoio social nos assentamentos investigados.

\section{Casos de Uso Abusivo de Álcool e Apoio Social}

Quanto ao AUDIT, do total de 1.492 indivíduos residentes nos assentamentos, aplicou-se a ferramenta de rastreio do uso de álcool em 767 , 
sendo 257 em Santo Antônio (49,03\% homens e $50,97 \%$ mulheres) e 510 em Canárias (42,75\% homens e $57,25 \%$ mulheres).

No geral, os resultados indicaram 89 casos identificados com possível consumo de risco, uso nocivo e dependente em ambos os assentamentos, o que vêm a corresponder a $11,67 \%$. A maioria está localizada em Canárias, sendo 55 homens e 16 mulheres, enquanto em Santo Antônio são 16 homens e 02 mulheres. Os homens se sobressaem às mulheres em termos de uso de álcool. Esse resultado já era esperado e indicado nos últimos levantamentos realizados pelo Instituto Nacional de Ciência e Tecnologia para Políticas Públicas do Álcool e outras Drogas (INPAD, 2012) e da Organização Pan Americana de Saúde (OPAS, 2015), embora indiquem um aumento no número de mulheres que ingerem bebidas alcoólicas.

Considerando as particularidades de cada assentamento, Canárias apresenta um maior consumo de álcool em comparação a Santo Antônio. Canárias é uma comunidade pesqueira, alvo de um estudo anterior que evidenciou uma estreita relação entre o uso do álcool e a rotina de trabalho, principalmente nas comunidades ribeirinhas, onde boa parte dos pescadores ingere bebidas alcoólicas para suportarem as condições de trabalho como o sol forte nos longos períodos da pesca em alto-mar. $\mathrm{O}$ trabalho solitário durante a noite na cata do caranguejo, assim como para suportar a infestação de mosquito no mangue e outras adversidades também justificam o consumo de álcool (Dimenstein et al., 2016).

Tabela 3

Distribuição em Relação ao Padrão de Uso de Álcool

\begin{tabular}{lcccccc}
\hline & \multicolumn{2}{c}{ Santo Antônio } & \multicolumn{2}{c}{ Canárias } & \multicolumn{2}{c}{ Total } \\
\cline { 2 - 7 } & $N^{\text {o }}$ & $\%$ & $N^{\text {o }}$ & $\%$ & $N^{\text {o }}$ & $\%$ \\
\hline Baixo Risco & 239 & 93 & 439 & 86.08 & 678 & 88.40 \\
Risco & 12 & 4.67 & 57 & 11.18 & 69 & 9 \\
Nocivo & 1 & 0.39 & 9 & 1.76 & 10 & 1.30 \\
Dependência & 5 & 1.95 & 5 & 0.98 & 10 & 1.30 \\
Total & 257 & 100 & 510 & 100 & 767 & 100 \\
\hline
\end{tabular}

No tocante à distribuição por padrão de uso, ilustrado na Tabela 3, em Santo Antônio, $4,67 \%$ da população faz uso de risco, seguindo por uso nocivo $(0,39 \%)$ e dependente $(1,95 \%)$. Em Canárias, há também o predomínio de uso prejudicial de risco $(11,18 \%)$, seguido por uso nocivo $(1,76 \%)$ e dependente $(0,98 \%)$. Cruzando os dados dos que apresentaram padrão de uso problemático de álcool com o perfil sócio demográfico, observa-se que são indivíduos com renda familiar de $1 / 2$ a 1 salário mínimo, possuem ensino fundamental incompleto/completo, casados, com filhos, jovens adultos, sendo pescadores e/ou agricultores. Contam com precárias condições de moradia, de renda, coleta pública de lixo, acesso à água encanada, à educação de qualidade e atendimento em saúde, produzindo impactos negativos no bem-estar e nos modos de vida das pessoas e da comunidade.

Em relação ao apoio social, aqueles que pontuaram no AUDIT acima do ponto corte $(\mathrm{n}=89)$ e responderam ao MOS-SSS, $82,1 \%$ são homens e $17,9 \%$ mulheres. A maioria dos respondentes com baixo apoio Afetivo são solteiros $(41,7 \%)$, enquanto os que contam com alto apoio são casados $(62,5 \%)$. A maioria com baixo apoio Material não possui filhos (55\%), enquanto com alto tem filhos $(56.80 \%)$. Na Tabela 4 pode-se observar os resultados do apoio social e padrão de uso de álcool. 
Tabela 4

Apoio Social (MOS-SSS) dos Respondentes do AUDIT - por Padrão de Uso

\begin{tabular}{|c|c|c|c|c|c|c|c|c|c|}
\hline \multirow{3}{*}{ Dimensão } & & \multicolumn{8}{|c|}{ AUDIT_categoria } \\
\hline & & \multicolumn{2}{|c|}{$\begin{array}{l}\text { Risco } \\
\text { (8 até } 15)\end{array}$} & \multicolumn{2}{|c|}{$\begin{array}{l}\text { Nocivo } \\
\text { (16 até } 19)\end{array}$} & \multicolumn{2}{|c|}{$\begin{array}{l}\text { Dependência } \\
\text { (20 ou mais) }\end{array}$} & \multicolumn{2}{|c|}{ Total } \\
\hline & & $N$ & $\%$ & $N$ & $\%$ & $N$ & $\%$ & $N$ & $\%$ \\
\hline \multirow{3}{*}{ Apoio Material } & $\begin{array}{l}\text { Abaixo da mediana } \\
\text { (Zero até } 95)\end{array}$ & 28 & 43.08 & 5 & 50 & 7 & 77.78 & 40 & 47.62 \\
\hline & $\begin{array}{l}\text { Acima da mediana } \\
\text { (acima de } 95)\end{array}$ & 37 & 56.92 & 5 & 50 & 2 & 22.22 & 44 & 52.38 \\
\hline & Total & 65 & 100 & 10 & 100 & 9 & 100 & 84 & 100 \\
\hline \multirow{3}{*}{ Apoio Afetivo } & $\begin{array}{l}\text { Abaixo da mediana } \\
\text { (Zero até } 95)\end{array}$ & 26 & 40 & 7 & 70 & 3 & 33.33 & 36 & 42.86 \\
\hline & $\begin{array}{l}\text { Acima da mediana } \\
\quad(\text { acima de } 95)\end{array}$ & 39 & 60 & 3 & 30 & 6 & 66.67 & 48 & 57.14 \\
\hline & Total & 65 & 100 & 10 & 100 & 9 & 100 & 84 & 100 \\
\hline \multirow{3}{*}{$\begin{array}{l}\text { Apoio } \\
\text { Emocional }\end{array}$} & $\begin{array}{l}\text { Abaixo da mediana } \\
\text { (Zero até } 90)\end{array}$ & 31 & 47.69 & 6 & 60 & 6 & 66.67 & 43 & 51.19 \\
\hline & $\begin{array}{l}\text { Acima da mediana } \\
\quad \text { (acima de } 90)\end{array}$ & 34 & 52.31 & 4 & 40 & 3 & 33.33 & 41 & 48.81 \\
\hline & Total & 65 & 100 & 10 & 100 & 9 & 100 & 84 & 100 \\
\hline \multirow{3}{*}{$\begin{array}{l}\text { Apoio } \\
\text { de Informação }\end{array}$} & $\begin{array}{l}\text { Abaixo da mediana } \\
\text { (Zero até } 90)\end{array}$ & 34 & 52.31 & 5 & 50 & 5 & 55.56 & 44 & 52.38 \\
\hline & $\begin{array}{l}\text { Acima da mediana } \\
\text { (acima de } 90)\end{array}$ & 31 & 47.69 & 5 & 50 & 4 & 44.44 & 40 & 47.62 \\
\hline & Total & 65 & 100 & 10 & 100 & 9 & 100 & 84 & 100 \\
\hline \multirow{3}{*}{$\begin{array}{l}\text { Apoio } \\
\text { de Interação } \\
\text { Positiva }\end{array}$} & $\begin{array}{l}\text { Abaixo da mediana } \\
\text { (Zero até 90) }\end{array}$ & 65 & 100 & 10 & 100 & 9 & 100 & 84 & 100 \\
\hline & $\begin{array}{l}\text { Acima da mediana } \\
\text { (acima de } 90)\end{array}$ & 0 & 0 & 0 & 0 & 0 & 0 & 0 & 0 \\
\hline & Total & 65 & 100 & 10 & 100 & 9 & 100 & 84 & 100 \\
\hline
\end{tabular}

Os resultados sugerem diferenças entre os sexos, pois enquanto os homens contam com alto apoio Material, Afetivo e Emocional, as mulheres percebem baixo apoio em todas as dimensões. Tal dado pode indicar que apesar de haver menos mulheres que fazem uso problemático de álcool em comparação aos homens, a questão de gênero é um elemento definidor de vulnerabilidade para as mulheres, visto que contam com menor apoio, associado ao estigma e maior grau de reprovação social existente na comunidade, já que ao beberem escapam do papel de boa mulher, cuidadora dos filhos, do marido e da casa (Alves, 2015). Para Zanello (2016), os papéis de gênero atribuídos, sobretudo, no casamento, constituem fator de proteção à saúde mental muito mais para os homens do que para mulheres. O cuidado com os filhos, marido, familiares, bem como a invisibilidade do trabalho doméstico tem levado muitas mulheres ao esgotamento físico e sofrimento psíquico. Ou seja, os atravessamentos 
de gênero impactam na percepção do apoio social recebido.

Os dados ainda sugerem que nos casos em que os padrões de consumo são mais problemáticos, observa-se menor percepção de apoio social em todas as dimensões. No uso de risco, por exemplo, observou-se apoio suficiente nas dimensões Material, Afetivo e Emocional, enquanto o Apoio de Informação foi insuficiente. Isso pode indicar certa dificuldade de acesso à orientação profissional na região. No uso nocivo, identificou-se baixo apoio em duas dimensões: Afetivo e Emocional. Entre aqueles que fazem uso dependente, observou-se baixo apoio em três dimensões: Material, Emocional e de Informação. E ao que se refere à Interação Positiva todos os respondentes estão abaixo da mediana quanto à oferta apoio, assim como nos dados TMC. Tais aspectos podem configurar um dado preocupante, pois talvez indique a necessidade de ações e estratégias de saúde que foquem no fortalecimento do vínculo e suporte social tanto da rede sócio familiar quanto comunitária desses indivíduos, bem como de ações de ampliação de divulgação de conhecimentos e informação sobre os problemas associados ao uso de álcool e possibilidades de cuidado. Miranda, Simpson, Azevedo, e Costa (2006) ressaltam a importância de um maior investimento da relação das equipes de saúde com os familiares na busca fortalecer as ações de cuidado, tendo em vista a família ser, muitas vezes, um fator protetivo frente aos casos de saúde mental. Mas, há situações em que é frequente encontrar relações familiares fragilizadas, assim como situações de perda de amizades e de relações profissionais.

As pessoas que fazem uso dependente apresentaram menor média de idade $(36,6$ anos, $D P=16)$, se comparado aos de uso de risco e nocivo (38,7 anos, $D P=13,6$; 44,1 anos, $D P=16,3$, respectivamente). Ou seja, trata-se de um grupo de pessoas jovens bastante vulnerável, que sugere contar com pouco apoio social. De forma preocupante, os levantamentos epidemiológicos têm registrado que as pessoas recorrem cada vez mais ao consumo do álcool de forma abusiva, bem como que esse consumo tem ocorrido cada vez mais cedo tanto para homens quanto para mulheres (OPAS, 2015).

\section{Considerações Finais}

Esse estudo indicou que as formas de adoecimento/sofrimento mental sugerem ter forte relação com as condições de vida, trabalho, saúde e educação da população, fortalecendo o campo de discussão sobre a Determinação Social da Saúde Mental, debate ainda tímido entre as equipes de saúde e formuladores de programas e políticas de saúde. Os casos identificados na pesquisa que envolvem transtornos mentais comuns e padrões problemáticos de consumo de álcool são ilustrativos desta possível relação, bem como o fato do apoio social pode estar condicionado a uma teia complexa de fatores. São aspectos que corroboram o estudo de Rodrigues e Madeira (2009) quando afirmam que o apoio social não apenas desempenha um papel importante na determinação dos níveis de saúde e bem-estar dos indivíduos, na busca de cuidados e adesão aos tratamentos, como também é determinado pelos contextos e condições de vida da população.

Por isso, destacamos a necessidade de desenvolver ações promocionais e preventivas em saúde (Estratégia Saúde da Família/ESF e Núcleo de Apoio à Saúde da Família/NASF), e o fortalecimento de vínculos sócio familiares em serviços como Centros de Referência da Assistência Social (CRAS) do Sistema Único da Assistência Social (SUAS). Tais equipes precisam fortalecer as redes de informação, de interação e de vivências comunitárias, apoiadas nos recursos do próprio território.

Por outro lado, a orientação das ações profissionais e dos serviços ofertados na Saúde e Assistência Social com base nas dimensões do apoio social, pode diminuir os níveis de vulnerabilidade e o impacto das questões de gênero e das condições de vida na saúde mental das populações assentadas, pois enquanto o adoecimento das mulheres indica relações com a dupla jornada de trabalho, com o número de filhos, com a violência de gênero, entre os homens, sobressai a sobrecarga de trabalho agrícola, a perda da vitalidade física e eventos estressores como separação, dentre outros. 


\section{Contribuições dos Autores}

Contribuição substancial no conceito e desenho do estudo: João Paulo Macedo, Magda Dimenstein.

Contribuição na coleta de dados: Brisana Índio do Brasil de Macêdo Silva, Hédina Rodrigues de Sousa, Ana Paula Almeida da Costa.

Contribuição na análise e interpretação de dados: (João Paulo Macedo, Brisana Índio do Brasil de Macêdo Silva, Hédina Rodrigues de Sousa, Ana Paula Almeida da Costa.

Contribuição para a preparação do manuscrito: João Paulo Macedo, Brisana Índio do Brasil de Macêdo Silva, Hédina Rodrigues de Sousa, Ana Paula Almeida da Costa.

Contribuição à revisão crítica, agregando conteúdo intelectual: João Paulo Macedo, Magda Dimenstein.

\section{Conflitos de interesse}

Os autores declaram não ter conflito de interesse relacionado à publicação deste manuscrito.

\section{Referências}

Abreu-Rodrigues, M. A., \& Seidl, E. M. F. (2013). Apoio social a pacientes coronarianos: Efeitos de intervenção comportamental com um familiar. Estudos de Psicologia (Campinas), 30(4), 619628. doi: 10.1590/S0103-166X2013000400015

Alves, T. M. (2015). A diversidade dos usos de substâncias psicoativas por mulheres em Parnaiba-PI a partir de suas histórias de vida: Uma perspectiva de gênero (Dissertação de mestrado, Universidade Federal do Piauí, Teresina, PI, Brasil).

Babor, T. F., Biddle-Higgins, J. C., Saunders, J. B., \& Monteiro, M. G. (2001). AUDIT: The Alcohol Use Disorders Identification Test: Guidelines for use in primary health care. Geneva: World Health Organization. Recuperado em http:// apps.who.int/iris/bitstream/10665/67205/1/ WHO_MSD_MSB_01.6a.pdf

Costa, A. G., \& Ludermir, A. B. (2005). Transtornos mentais comuns e Apoio social: Estudo em comunidade rural da Zona da Mata de Pernambuco, Brasil. Cadernos de Saúde
Pública, 21(1), 73-79. doi: 10.1590/ S0102311X2005000100009

Dimenstein, M. D. B., Leite, J. F., Macedo, J. P. S., \& Dantas, C. M. B. (Eds.). (2016). Condições de vida e saúde mental em contextos rurais. São Paulo, SP: Intermeios.

Gonçalves, D. M., Stein, A. T., \& Kapczinski, F. (2008). Avaliação de desempenho do SelfReporting Questionnaire como instrumento de rastreamento psiquiátrico: Um estudo comparativo com o Structured Clinical Interview for DSM-IV-TR. Cadernos de Saúde Pública, 24(2), 380-390. doi: 10.1590/S0102311X2008000200017

Griep, R. H., Chor, D., Faerstein, E., Werneck, G. L., \& Lopes, C. S. (2005). Validade de constructo de escala de apoio social do Medical Outcomes Study adaptada para o português no Estudo Pró-Saúde. Caderno de Saúde Pública, 21(3), 703714. doi: 10.1590/S0102-311X2005000300004

Instituto Brasileiro de Geografia e Estatística. (2010). Cidades. Recuperado em http://www.cidades. ibge.gov.br/xtras/home.php

Instituto Nacional de Ciência e Tecnologia para Políticas Públicas do Álcool e outras Drogas. (2012). II Levantamento Nacional de Álcool e Outras Drogas - LENAD. São Paulo, SP: Autor.

Jussani, N. C., Serafim, D., \& Marcon, S. S. (2007). Rede social durante a expansão da família. Revista Brasileira de Enfermagem, 60(2), 184-189. doi: 10.1590/S0034-71672007000200011

Loureiro, A., Lima, J., Partidário, M. R., \& Santana, P. (2015). Condicionantes de saúde mental e os instrumentos de avaliação de impactos. In P. Santana (Ed.), Territórios e saúde mental em tempos de crises (pp. 11-27). Portugal: Universidade de Coimbra. doi: 10.14195/978989-26-1105-1

Mari, J. J., \& Williams, P. A. (1986). Validity study of a psychiatric screening questionnaire (SRQ20) in primarycare in the city of São Paulo. British Journal of Psychiatry, 148, 23-26.

Miranda, N., Simpson, A., Azevedo, M., \& Costa, S. (2006). O impacto negativo dos transtornos do uso e abuso do álcool na convivência familiar. Revista Eletrônica de Enfermagem, 8(2), 222232.

Moretti-Pires, R. O., \& Corradi-Webster, C. M. (2011). Adaptação e validação do Alcohol Use 
Disorder Identification Test (AUDIT) para população ribeirinha do interior da Amazônia, Brasil. Cadernos de Saúde Pública, 27(3), 497-509. doi: 10.1590/S0102-311X2011000300010

Organização Pan Americana de Saúde. (2015). Informe sobre la situación regional sobre el alcohol y la salud en las Américas. Washington, DC: Autor.

Peña, R. F. (2005). Redes Sociales, apoyo social y salude. Periferia, 3, 1-16. Recuperado em http:// revista-redes.rediris.es/Periferia/english/number3/periferia_3_3.pdf

Pinho, P. S., \& Araújo, T. M. (2012). Associação entre sobrecarga doméstica e transtornos mentais comuns em mulheres. Revista Brasileira de Epidemiologia, 15(3), 560-572. doi: 10.1590/ S1415-790X2012000300010

Programa das Nações Unidas para o Desenvolvimento. (2010). Relatório de Desenvolvimento Humano 2010- A verdadeira riqueza das nações: Vias para o desenvolvimento humano. New York: Autor.

Rabasquinho, C., \& Pereira, H. (2007). Gênero e Saúde Mental: Uma abordagem epidemiológica. Análise Psicológica, 25(3), 439-454.

Rehm, J., \& Parry, C. (2009). Alcohol consumption and infectious diseases in South Africa. Lancet, 374(9707), 2053. doi: 10.1016/S01406736(09)62150-4

Rocha, M. L. A., Guimarães, M. B. L., \& Cunha, M. B. (2012). O processo de recuperação do uso indevido de drogas em igrejas pentecostais Assembleia de Deus. Interface- Comunicação Saúde e Educação, 16(40), 177-190.

Rodrigues, J. S. M., Ferreira, N. M. L. A., \& Caliri, M. H. L. (2013). Caracterização do apoio social percebido pela família do doente adulto com câncer. Medicina (Ribeirão Preto), 46(3), 289296.
Rodrigues, V., \& Madeira, M. (2009). Suporte social e saúde mental. Revisão da Literatura. Revista da Faculdade de Ciências da Saúde (Porto), 6, 390-399. Recuperado em http://bdigital.ufp.pt/ bitstream/10284/1293/2/390-399_FCS_06_-6. pdf

Ronzani, T. M. (2008). Padrão de uso de álcool entre Atenção Primária à Saúde: Estudo comparativo. Revistade APS, 11(2), 163-171.

Santana, P. (Ed.). (2015). Territórios e saúde mental em tempos de crises. Portugal: Universidade de Coimbra. doi: 10.14195/978-989-26-1105-1

Santos, M. N. (2015). Solidão e saúde de idosos institucionalizados (Dissertação de mestrado, Faculdade de Psicologia, Universidade de Lisboa, Portugal).

Sherbourne, C. D., \& Stewart, A. L. (1991). The MOS social support survey. Social Science \& Medicine, 32(6), 705-714. Retrieved from https://www.ncbi.nlm.nih.gov/pubmed/2035047

Silva, A. C. (2015). Levantamento do padrão de consumo de tabaco e outras drogas na gestão de cuidados em saúde em assentados de uma comunidade rural (Dissertação de mestrado, Universidade Federal de Goiás, Catalão, GO, Brasil).

Sparovek, G. (2003). A qualidade dos assentamentos de reforma agrária brasileira. São Paulo, SP: Páginas \& Letras.

Zanello, V. (2016). Saúde mental, gênero e dispositivo. In M. D. B. Dimenstein, J. F. Leite, J. P. S. Macedo, \& C. M. B. Dantas (Eds.), Condições de vida e saúde mental em contextos rurais. São Paulo, SP: Intermeios.

Recebido: $15 / 03 / 2017$

$1^{a}$ revisão: 18/10/2017

Aceite final: 19/10/2017

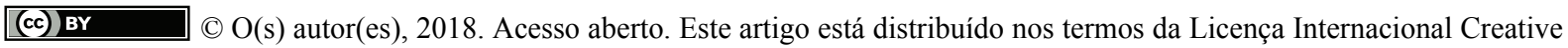
Commons Atribuição 4.0 (http://creativecommons.org/licenses/by/4.0/), que permite o uso, distribuição e reprodução sem restrições em qualquer meio, desde que você dê crédito apropriado ao(s) autor(es) original(ais) e à fonte, fornecer um link para a licença Creative Commons e indicar se as alterações foram feitas. 